\title{
Is the Prevalence of Coronary Artery Anomalies Different in Countries with Small Populations? - A Retrospective Study in Northern Cyprus
}

\author{
Musa Muhtaroglu, ${ }^{1}$ Sevda Lafc1 Fahrioglu, ${ }^{2}$ Osman Beton, ${ }^{3,4}$ Sezgin İlgi ${ }^{1}$ \\ ${ }^{1}$ Departments of Anatomy, Near East University Faculty of Medicine, Nicosia, Cyprus; ${ }^{2}$ Departments of Anatomy, Cyprus \\ International University Faculty of Medicine, Nicosia, Cyprus; ${ }^{3}$ Department of Cardiology, Cyprus International University Faculty \\ of Medicine, Nicosia, Cyprus; ${ }^{4}$ Departments of Cardiology, Dr. Burhan Nalbantoğlu State Hospital, Nicosia, Cyprus
}

\section{ABSTRACT}

Background: Although the prevalence of coronary artery anomalies (CAA) is due to accidental and rare discoveries, it varies between different countries or geographies. CAA are rare congenital disorders having various clinical definitions. Its prevalence varies in angiographic and autopsy series in adult populations and is approximately $1 \%$ in average. While the incidence ranges from $0.2 \%$ to $5.64 \%$ in coronary angiographic (CAG) studies, it is around $0.3 \%$ in autopsy series. We aimed to estimate the frequency of CAA in our patient population.

Methods: The coronary angiographic data of 4099 consecutive adult patients, who underwent CAG between January 2019 and December 2020, were analyzed and retrospectively studied.

Results: The mean age of the total patients who underwent CAG was $61.59 \pm 13.67$ years (range, 18-98 years). CAA were found in 76 patients $(1.85 \%$ incidence), origin and course anomaly in 62 patients $(81.6 \%)$, and coronary artery termination anomaly in 14 patients $(18.4 \%)$. Separate exits of the left anterior descending (LAD) and left circumflex (LCX) coronary artery from the left sinus of Valsalva (LSV) were the most common anomalies (36.84\%). Coronary artery fistulas were seen in $14(18.42 \%)$ patients. Abnormal origin of left circumflex artery (LCX) from the right coronary artery (RCA) or right sinus valsalva (RSV) was seen in 13 (17.11\%) patients. Outflow anomalies from the contralateral coronary sinus were detected in $10(13.16 \%)$ patients.

Conclusion: The incidence and pattern of CAA in our patient population showed similarity with previous studies. Physicians should be aware of CAA that may be associated with potentially serious cardiac incidents, because recognition of these CAA is important for the decision of treatment procedures.

Received August 5, 2021; accepted September 14, 2021.

Correspondence: Musa Mubtaroglu; Anatomy Department, Faculty of Medicine, Near East University, Nicosia, Cyprus, Telephone +905428540684 (e-mail: musamubtaroglu@botmail.com).

\section{INTRODUCTION}

Congenital coronary artery anomalies (CAA) are the modifications of their origin, course or structure, and their incidence ranges from $0.2 \%$ to $5.64 \%$ of the general population [Kashyap 2021; Sidhu 2019; Yamanaka 1990; Angelini 2002]. Although the prevalence of CAA is due to accidental and rare discoveries, it varies between different countries or geographies [Jiang 2021; Sidhu 2019]. Although the majority are asymptomatic, it is the second leading cause of sudden deaths in young athletes. CAAs are a diverse group of congenital disorders with highly variable symptoms and pathophysiological mechanisms. They are found congenitally, but relatively few are symptomatic. Although generally benign, some CAAs are associated with more serious clinical outcomes, such as congestive heart failure, arrhythmia, myocardial infarction, syncope, and sudden death [Kashyap 2021; Yuksel 2013]. CAG traditionally has been used for detailing coronary vessels before intervention and surgery and remains the reference standard of imaging modality [Jiang 2021; Almeida 2020]. On the other hand, computed tomographic angiography is a noninvasive imaging approach that is safe and effective in finding coronary anomalies [Yildiz 2010; Budoff 2006]. Furthermore, the computed tomographic angiography provides detailed anatomic information through 3-dimensional imaging, which may be difficult to obtain through CAG. Although CAG has some natural disadvantages, its availability, high accuracy and low risk have made it a useful tool for diagnosing coronary artery disease (CAD). The recognition of these anomalies is particularly important when a patient requires percutaneous coronary intervention or heart surgery [ $\mathrm{Li}$ 2015; Jiang 2021]. Based on the vascular supply of posterior interventricular septum, coronary artery dominance phenotypes may occur as left dominance (LD), right dominance (RD), and co-dominance (CD) [Gorlin 1976; Leaman 1981]. The origin of the posterior descending artery (PDA) is the main determinant for LD, RD, and CD [Kuno 2013; Bazzocchi 2011; Vasheghani 2008]. When poster lateral branches supply most of the left ventricular myocardial and when the PDA originates from the LCX artery, the case is classified as LD [Eyuboglu 2016]. RD cases have posterior interventricular septum (IVS) supplied by the posterior descending branch of the RCA. When the IVS is shared by RCA and LCX, the case is labelled as CD [Moriwaki 2017]. In this study, we 
examined CAG in detail to determine the anomalies of origin, course, and termination of the coronary artery, as well as the dominance of the coronary arteries in our hospital. There are several classifications for coronary artery anomalies. We preferred to examine the anomalies of origin, course and termination of coronary arteries, which is one of the classifications modified for use in clinic and practice, by dividing them into two groups [Liberthson 1974; Chu 1993; Kardos 1997; Yildiz 2010; Turkmen 2013].

\section{MATERIALS AND METHODS}

Our study was carried out to determine the incidence and prevalence of coronary artery origin course and termination anomalies by retrospectively evaluating 4376 coronary angiography reports made in Dr. Burhan Nalbantoğlu State Hospital Cardiology Service between January 2019 and December 2020. When the patients with more than one CAG report were reduced from the total coronary angiography (CAG) number to single, a total of 4099 different patients were identified, so the evaluations were made on this number. Angiograms were reviewed by an experienced independent cardiologist and two anatomists, who agreed on the origin, course, and termination of abnormal coronary arteries. Patients with coronary anomalies associated with complex congenital heart disease were not included in this study. To calculate the total incidence of coronary artery anomalies, the percentage of patients with coronary artery anomalies in 4099 cases undergoing CAG was found. Based on the total number of patients with CAAs, the percentage distribution of different types of CAAs was calculated, and the prevalence of each type of CAA among all patients who underwent CAG, the mean age of the patients, and the female/male ratio were determined. In addition, the coronary artery dominance pattern of all patients who underwent CAG was examined and recorded one by one. The statistical package for the social sciences (SPSS software Version 23.0, SPSS, Inc., Chicago, IL, USA) was used to analyze the data. Categorical variables are presented as percent (\%), frequencies and continuous variables are presented as mean \pm standard deviation (SD).

Coronary angiography procedure: After the history, physical examination and laboratory tests of the CAG patients were evaluated, the patients diagnosed with CAG indication were informed about CAG. After explaining the examination to be performed and the benefits and risks of this examination to the patient, written consent was obtained. Electrocardiogram, serum electrolytes and creatinine, blood count and coagulation parameters were repeated 16-24 hours before the procedure. The medical treatment that the patient was receiving was continued before the procedure. Patients with consents and no contraindications were taken to the catheterization laboratory for diagnosis and treatment. Catheterization was performed while the patient was awake and, if necessary, with sedation. The area to be entered into the vascular access was sterilized and anesthetized with local anesthesia ( $2 \%$ prilocaine hydrochloride). The procedure was generally performed with the seldinger method, which is the most commonly used percutaneous needle-guidewiredilator technique. Catheterization was applied mostly from the right femoral artery, less from the left femoral artery and brachial artery. When performing selective CAG, usually right and left judkins (JR 3.5, 4, 5, 6 and JL 3.5, 4, 4.5, 5 and 6), more rarely Amplatz (AR I, II, III and AL I, II, III) and Multipurpose (A, B, C) catheters, pigtail catheter used for left heart catheterization and aortography. Radiopaque agents with low allergic and nephrotoxic properties (low osmolarity and non-ionic) were preferred for imaging the coronary arteries. Images were taken in at least four projections for the left coronary system and at least two projections for the right coronary system and stored in digital memory. Left heart catheterization was performed in all patients with suspected coronary artery anomaly and normal renal functions. In some cases, aortography was performed in addition to these procedures. Selective CAG, left ventriculography, and aortography were performed after measuring hemodynamic parameters and blood oxygen saturation in patients with heart valve disease or congenital heart disease.

\section{RESULTS}

CAA was detected in 76 patients in 4099 CAG examinations ( $1.85 \%$ incidence). Of 76 patients ( 50 men, 26 women), 62 patients $(81.6 \%)$ had anomaly of origin and distribution, and 14 patients $(18.4 \%)$ had coronary artery fistula (CAF). (Table 1) The mean age of 76 patients was $62.97 \pm 12.48$ years (age range, 29-82 years). The main characteristics of the patient population categorized, according to coronary artery predominance in this study, are shown in Table 1 . Of 4099 patients, 2994 (73\%) were found to have right coronary artery dominance, $693(16.9 \%)$ left circumflex artery dominance, and $412(10.1 \%)$ were found to be co-dominance type. The most common anomaly types in our study are, respectively, absence of LMCA in $36.84 \%$, coronary artery fistulas in $18.42 \%$, origination of LCX artery from RCA and RSV with $17.11 \%$, exit from opposite coronary sinus in $13.16 \%$, abnormal exit from appropriate sinus valsalva in $7.89 \%$, and $5.26 \%$ isolated single coronary artery in 4 patients. (Table 3) (Table 4) In our study, the findings of coronary anomalies

Table 1. Distribution of patients according to the presence of coronary anomaly and coronary dominance

\begin{tabular}{lcc}
\hline Total angiography & $N=4099$ & $\%$ \\
\hline Total anomaly & 76 & - \\
Origin and course anomalies & 62 & 81.6 \\
Coronary artery termination anomalies & 14 & 18.4 \\
Coronary dominance & & \\
RCA dominance & 2994 & 73 \\
LCX dominance & 693 & 16.9 \\
Co-dominance & 412 & 10.1
\end{tabular}


Table 2. Distribution of anomaly types of patients by age and gender

\begin{tabular}{lccccccccccc}
\hline & \multicolumn{4}{c}{ Female } & \multicolumn{3}{c}{ Male } & Total \\
& $N$ & $\%$ & Age (SD) & $N$ & $\%$ & Age (SD) & $N$ & Age (SD) & R (\%) \\
\hline Total anomaly & 26 & 34.2 & $67.57(11.80)$ & 50 & 65.8 & $60.58(12.26)$ & 76 & $62.97(12.48)$ & 1.85 \\
Origin and course anomalies & 19 & 30.6 & $72.9(9.95)$ & 43 & 69.4 & $59.63(13.45)$ & 62 & $62.66(12.53)$ & 1.51 \\
Coronary artery termination anomalies & 7 & 50 & $64.29(13.78)$ & 7 & 50 & $64.43(12.47)$ & 14 & $59.30(12.96)$ & 0.34 \\
\hline
\end{tabular}

SD, standard deviation; R, ratio (\%)

Table 3. Age, gender and incidence of the types of origin and course anomalies within themselves

\begin{tabular}{|c|c|c|c|c|c|c|c|c|c|}
\hline & $N$ & $\%$ & Age (SD) & $N$ & $\%$ & Age (SD) & $N$ & Age (SD) & $\mathrm{R} / \mathrm{RwA}(\%)$ \\
\hline Abnormal origin from appropriate sinus of Valsalva & 2 & 33.3 & $72.50(2.12)$ & 4 & 66.7 & $50.75(2.63)$ & 6 & $58(11.45)$ & $0.15 / 7.89$ \\
\hline Abnormal origin from normal coronary sinuses & 0 & - & - & 1 & 100 & 72 & 1 & 72 & $0.02 / 1.31$ \\
\hline Origin of RCA from LSV & 1 & 14.3 & 80 & 6 & 85.7 & $65.89(13.1)$ & 7 & $66.85(15.29)$ & $0.17 / 9.21$ \\
\hline Origin of LAD from RSV & 0 & 0.00 & - & 0 & 0.00 & - & 0 & - & - \\
\hline Origin of CX from RCA or RSV & 4 & 30.8 & $54.50(8.74)$ & 9 & 69.2 & $58(12.17)$ & 13 & $56.92(10.98)$ & $0.31 / 17.11$ \\
\hline Origin of LMCA from RSV & 1 & 33.3 & 65 & 2 & 66.7 & $48(5.66)$ & 3 & $53.66(10.59)$ & $0.07 / 3.94$ \\
\hline Single coronary artery & 0 & 0 & - & 4 & 100 & $60.50(10.41)$ & 4 & $60.50(10.41)$ & $0.09 / 5.26$ \\
\hline
\end{tabular}

SD, standard deviation; R, ratio (\%); RwA, ratio within anomalies (\%)

Table 4. Age, gender and incidence of the types of coronary artery termination anomalies within themselves

\begin{tabular}{|c|c|c|c|c|c|c|c|c|c|}
\hline & $N$ & $\%$ & Age (SD) & $N$ & $\%$ & Age (SD) & $N$ & Age (SD) & $\mathrm{R} / \mathrm{RwA}(\%)$ \\
\hline LAD-PA fistula & 7 & 50 & $64.29(13.78)$ & 7 & 50 & $64.43(12.47)$ & 14 & $64.35(12.62)$ & $0.34 / 18.42$ \\
\hline Sinusoid coronary artery connections & 0 & 0.00 & - & 0 & 0.00 & - & 0 & - & - \\
\hline
\end{tabular}

SD, standard deviation; R, ratio (\%); RwA, ratio within anomalies (\%)

were incidental.

\section{DISCUSSION}

Coronary artery anomalies (CAA) are rare congenital disorders with various clinical presentations. Its prevalence in the adult population varies in angiographic and autopsy series and averages around $1 \%$. The incidence ranges from
$0.2 \%$ to $5.64 \%$ in angiographic studies, while it is around $0.3 \%$ in autopsy series [Yamanaka 1990; Ciesunski 1993; Kashyap 2021]. Similarly, we found the incidence of coronary artery anomaly to be $1.85 \%$ in patients who underwent coronary angiography for diagnosis. Based on the vascular supply of the posterior interventricular septum, coronary artery dominance phenotypes may occur as left dominance (LD), right dominance (RD), and co-dominance $(\mathrm{CD})$. The origin of the posterior descending artery 
is the main determinant for $\mathrm{LD}, \mathrm{RD}$, and $\mathrm{CD}$ [Gorlin 1976; Leaman 1981; Peng 2018]. RD makes up the majority of the cases with a prevalence of $82 \%$ to $89 \%$ in the general population. Prevalence of $\mathrm{LD}$ is $5 \%$ to $12 \%$, and the prevalence of CD is 3\% to 7\% [Angelini 2002; Knaapen 2013]. In the current study, we observed 2994 of the 4099 patients as $\mathrm{RD}$, which indicates a ratio of $73 \% ; 693$ patients as $\mathrm{LD}$, which comes to a ratio of $16.9 \%$; and 412 patients as CD, which comes to a ratio of $10.1 \%$. Thus, our results are similar to prior results reported in the literature. The limitation of our study was that only patients undergoing coronary angiogram were included, rather than a randomly selected sample from the entire population. CAAs can lead to lifethreatening symptoms, such as arrhythmias, syncope, myocardial infarction, or sudden death [Angelini 2002; Kashyap 2021]. In one of two studies conducted, a prior history of angina or syncope was reported in $33 \%$ of patients with abnormal coronary anatomy, and in the other in $31 \%$ [Yildiz 2010; Yamanaka 1990]. Thirty-six percent of patients in our study population had typical cardiovascular symptoms. Coronary artery origin and course anomalies were found to be $81.6 \%$ and coronary artery fistulas $18.4 \%$ in our study, compared with previous similar studies, the prevalence of origin and course anomalies was lower and coronary artery fistulas were found to be higher [Yamanaka 1990; Kardos 1997; Aydinlar 2005; Yildiz 2010; Turkmen 2013]. Origin of LAD and LCX from ostia separate from the left sinus of Valsalva (LSV), absence of LMCA (36.84\%) were the most common anomalies in our study group and were similar to previous studies [Yildiz 2010; Yamanaka 1990; Aydinlar 2005; Turkmen 2013]. Coronary artery fistula (CAF) was the second common anomaly group with $(18.42 \%)$ in our study. Coronary artery fistulas (CAFs) are rare defects in the coronary circulation system. They are congenital or acquired malformations in the assembly and circuitry of the coronary arteries. CAFs can be divided into two broad categories. Coronary-cameral fistulas are abnormal connections between the coronary arteries and any of the heart chambers. Coronary arteriovenous malformations are abnormal connections between coronary arteries and portions of systemic/pulmonary circulation vessels. Although most coronary artery fistulas are diagnosed incidentally during coronary catheterization, some patients with this disease may present with signs and symptoms of congestive heart failure, myocardial infarction, pulmonary hypertension, and other cardiopulmonary functional abnormalities. Regarding the diagnosis, coronary angiography and coronary computed tomography angiography (CTA) are considered quite reliable in the diagnosis of CAFs. Catheterized closure is often stated as the main modality of treatment [Qureshi 2006; Rao 2021]. There are three main causes of CAFs. Most commonly, CAFs occur congenitally as a result of abnormal embryological development. The most common form of acquired CAFs is caused by trauma, such as gunshot wounds or stab wounds. Iatrogenic causes of CAFs include interventional cardiac procedures, such as coronary artery bypass grafting, cardiac angiography, valve replacements, device implants, or biopsies [Rao 2021; Sunkara 2017]. The incidence is approximately $0.002 \%$ in the general population and $0.3 \%$ to $0.4 \%$ in patients with congenital heart defects, although more and more studies are being conducted on the prevalence of CAFs, the prevalence was found to be $0.9 \%$ in one study [Yildiz 2010; Lim 2014; Maleszka 2005]. CAFs can lead to various cardiopulmonary functional abnormalities. The severity of the presenting symptoms depends on the source of the fistula, area of entry, and length of the fistula, as well as the volume of blood shunted. The most common origin is the RCA, followed by LMCA. The most common insertions (from most common to least common) are the right ventricle, right atrium, and pulmonary arteries. However, more recent studies have shown that generally, the most common fistula is between the LMCA or the left anterior descending artery (LAD) and pulmonary arteries (PA) [Lim 2014; Maleszka 2005; Verdini 2016]. In our study, the most common fistula was detected between the LAD and PA, which was similar to results in the literature [Turkmen 2013; Yildiz 2010; Yamanaka 1990; Aydinlar 2005; Verdini 2016]. We estimate that the high incidence of CAFs (0.34\%) in our study was due to the low patient population. After CAFs anomalies in a total of $13(17.11 \%)$ patients (incidence $0.31 \%$ ), 4 women with a mean age of $54.50 \pm 8.74$ years and nine men with a mean age of $58 \pm 12.17$ years, LCX artery anomaly originating from RSV and RCA was detected. The incidence of LCX originating from RCA and RSV is generally believed to be of no clinical significance, due to the dorsal course of the left ventricle [Budoff 2006]. In our study, anomaly of origin from the contralateral coronary sinus was detected in $10(13.6 \%)$ patients. LMCA or LAD from the RSV and RCA from the LSV are clinically important as they are associated with sudden cardiac death [Yildiz 2010; Budoff 2006; Liberthson 1996]. In our study population, there were 10 patients with anomaly of the contralateral sinus of valsalva. We found a total of 7 $(0.17 \% / 9.21 \%)$ patients, an 80 -year-old woman and six men with a mean age of $65.89 \pm 13.1$ years, with RCA anomaly originating from the LSV. The incidence of RCA originating from LSV or LAD was reported between $8 \%, 8.9 \%$, and $16 \%$ in three different studies similar to our study, two of which were from Turkey [Yildiz 2010; Yamanaka 1990; Cohen 2002]. Out of 4099 cases, the anomaly of LMCA originating from the RSV was detected in three $(0.07 \% / 3.94 \%)$ patients, a 65 -year-old woman and two men with a mean age of $48 \pm 5.66$. Abnormal origin of the LMCA from the RSV is a rare congenital coronary anomaly [Yildiz 2010; Yamanaka 1990; Liberthson 1974; Wilkins 1988]. The incidence of LMCA originating from RSV was found to be $0.017 \%$ in a large-scale study of 126,595 patients by Yamanaka and Hobbs [Yamanaka 1990]. LMCA, $\mathrm{LAD}$, and RCA may originate from the pulmonary artery, although the incidence rate is low. Unfortunately, about $90 \%$ of patients with these anomalies die in infancy [Yildiz 2010; Aydinlar 2005]. Abnormal origins of the coronary arteries from the appropriate sinus are less common anomalies. Difficulties are experienced in the classification of these anomalies, since there are no clear measurement 
criteria. The incidence of this anomaly in studies varies between $0.3 \%$ and $0.7 \%$ [Menke 1985]. In our study, this rate was found to be $0.15 \%$. It has been reported in the literature that the risk of atherosclerosis increases in the ostia of high and low origin arteries. Cases claimed to cause myocardial feed deterioration and sudden death have been reported, especially due to the slit-like ostia of the highoutput arteries and the absence of sinuses that would allow blood to pool in diastole and be directed to the coronary arteries [Menke 1985]. Inappropriate origins of coronary arteries are benign anomalies, but they usually cause prolonged CAG time and difficulty in the procedure, due to the lack of expected localization of the ostia. In the single coronary artery anomaly type, all coronary arteries originate from a single ostium as a single common trunk [Montaudon 2007]. This artery feeds the whole heart [Earls 2006]. The course of a single coronary artery can be followed with many different variations [Lipton 1976; Earls 2006]. A single coronary artery can be divided into two and follow the distribution of the right and left coronary arteries, can follow the course of the right or left coronary artery alone, or show a completely different distribution from the course patterns of normal coronary vasculature [Lipton 1976]. It does not show any clinical manifestation in the absence of other concomitant coronary artery disease or a cardiac-dependent malformation [Lipton 1976]. Potential clinical manifestations include cardiac ischemia, cardiomyopathy, and congestive heart failure [Lipton 1976]. These presentations are largely due to the interarterial course of a major artery [Lipton 1976]. This may play a role in sudden death [Lipton 1976]. In these cases, surgical treatments with coronary artery bypass are recommended [Erez 2006]. The incidence of single coronary artery anomaly is $0.026-$ $0.4 \%$ in the population and constitutes $<3 \%$ of congenital coronary anomalies without gender preference [Conkbayir 2019; Yaymaci 2010; Desmet 1992]. In our study, a single coronary artery anomaly $(0.09 \%)$ was found in four male patients with a mean age of $60.50 \pm 10.41$ years, and the incidence was similar to those in other studies.

\section{CONCLUSIONS}

CAA is more common in large centers and in their daily practice. The incidence of anomalies may vary between different countries and geographies. CAAs, although usually asymptomatic, are usually isolated lesions not associated with congenital heart disease or atherosclerosis, some of which can cause anginal symptoms, myocardial infarction, and sudden death. Recognition of coronary anatomical patterns, variations, and coronary artery anomalies is important for the procedure to be applied, especially in patients scheduled for coronary angioplasty or cardiac surgery. Further prospective studies are needed to assess the incidence and prognosis of CAA. Future studies may utilize computed tomographic angiography as a noninvasive and safe alternative to define high risk coronary anomalies, such as those having an intra-arterial course that may put the patient in increased risk of sudden cardiac death.

\section{REFERENCES}

Almeida AS, Fuchs SC, Fuchs FC, et al. 2020. Effectiveness of clinical, surgical and percutaneous treatment to prevent cardiovascular events in patients referred for elective coronary angiography: an observational study. Vascular Health and Risk Management. 16:285-297.

Angelini P, Velasco JA, Flamm S. 2002. Coronary anomalies: incidence, pathophysiology, and clinical relevance. Circulation. 105:2449-54.

Aydinlar A, Çiçek D, Sentürk T, et al. 2005. Primary Congenital Anomalies of the Coronary Arteries A Coronary Arteriographic Study in Western Turkey. International heart journal. 46:97-103.

Bazzocchi G, Romagnoli A, Sperandio M, et al. 2011. Evaluation with 64-slice CT of the prevalence of coronary artery variants and congenital anomalies: a retrospective study of 3,236 patients. La Radiol Med. 116:675-89.

Budoff MJ, Ahmed V, Gul KM, Mao SS, Gopal A. 2006. Coronary anomalies by cardiac computed tomographic angiography. Clinical Cardiology: An International Indexed and Peer-Reviewed Journal for Advances in the Treatment of Cardiovascular Disease. 29: 489-93.

Chu E, Cheitlin MD. 1993. Diagnostic considerations in patients with suspected coronary artery anomalies. Am Heart J. 126: 1427-1438.

Ciesunski G, Rapprich B, Kober G. 1993. Coronary anomalies: incidence and importance. Clinical cardiology. 16:711-5.

Cohen MG, Tolleson TR, Peter RH, Harrison JK, Sketch Jr MH. 2002. Successful percutaneous coronary intervention with stent implantation in anomalous right coronary arteries arising from the left sinus of valsalva: a report of two cases. Catheterization and cardiovascular interventions. 55: $105-8$

Conkbayir C, Coskun U, Oztas DM, et al. 2019. Coronary Arteries Arising from Single Coronary Ostium: A Case Report. Heart Surg Forum. 22:E269-E270.

Desmet W, Vanhaecke J, Vrolix M, et al. 1992. Isolated single coronary artery: a review of 50,000 consecutive coronary angiographies Eur Heart J. 13:1637-40.

Earls JP. 2006. Coronary artery anomalies. Techniques in vascular and interventional radiology. 9: 210-7.

Erez E, Tam VK, Doublin NA, Stakes J. 2006. Anomalous coronary artery with aortic origin and course between the great arteries: improved diagnosis, anatomic findings, and surgical treatment. The Annals of thoracic surgery. 82:973-7.

Eyuboglu M, Ekinci MA, Karakoyun S, et al. 2016. Fragmented QRS for risk stratification in patients undergoing first diagnostic coronary angiography. Arq Bras Cardiol. 107:299-304.

Jiang X, Zhou P, Wen C, et al. 2021. Coronary Anomalies in 11,267 Southwest Chinese Patients Determined by Angiography. BioMed Research International. 6693784.

Kardos A, Babai L, Rudas L, et al. 1997. Epidemiology of congenital coronary artery anomalies: a coronary arteriography study on a central European population. Catheterization and cardiovascular diagnosis. 42:270-5.

Kashyap JR, Kumar S. 2021. Prevalence and Pattern of Congenital Coronary Artery Anomalies in Patients Undergoing Coronary Angiography at a Tertiary Care Hospital of Northern India. Cureus. 13:e14399.

Knaapen M, Koch AH, Koch C, et al. 2013. Prevalence of left and balanced coronary arterial dominance decreases with increasing age of patients at autopsy. A postmortem coronary angiograms study. Cardiovasc 
Pathol. 22:49-53.

Kuno T, Numasawa Y, Miyata H, et al. 2013. Impact of coronary dominance on in-hospital outcomes after percutaneous coronary intervention in patients with acute coronary syndrome. PLoS One. 8:e72672.

Liberthson RR. 1996. Sudden death from cardiac causes in children and young adults. New England Journal of Medicine. 334:1039-44.

Liberthson RR, DINSMORE RE, Bharati S, et al. 1974. Aberrant coronary artery origin from the aorta: diagnosis and clinical significance. Circulation. 50:774-9.

Lim JJ, Jung JI, Lee BY, Lee HG. 2014. Prevalence and types of coronary artery fistulas detected with coronary CT angiography. American Journal of Roentgenology. 203:W237-43.

Lipton MJ, Barry WH, Obrez I, Silverman JF, Wexler L. 1979. Isolated single coronary artery: diagnosis, angiographic classification, and clinical significance. Radiology. 130:39-47.

Maleszka A, Kleikamp G, Minami K, Peterschröder A, Körfer R. 2005. Giant coronary arteriovenous fistula. Zeitschrift für Kardiologie. 94:38-43.

Menke DM, Waller BF, Bless JE. 1985. Hypoplastic coronary arteries and high takeoff position of the right coronary ostium: a fatal combination of congenital coronary artery anomalies in an amateur athlete. Chest. 88: 299-301.

Montaudon M, Latrabe V, Iriart X, Caix P, Laurent F. 2007. Congenital coronary arteries anomalies: review of the literature and multidetector computed tomography (MDCT)-appearance. Surgical and Radiologic Anatomy. 29: 343-55.

Moriwaki K, Dohi K, Omori T, et al. 2017. A survival case of fulminant right-side dominant eosinophilic myocarditis. Int Heart J. 58:459-62.

Peng L, Guo X, Gao Y, et al. 2018. Impact of right coronary dominance on triple-vessel coronary artery disease: A cross-sectional study. Medicine (Baltimore). 97:e11685.

Qureshi SA. 2006. Coronary arterial fistulas. Orphanet Journal of Rare Diseases. 1:1-51.
Rao SS, Agasthi P. 2021. Coronary Artery Fistula. StatPearls [Internet]. Treasure Island FL: StatPearls Publishing.

Sidhu NS, Wander GS, Monga A, Kaur A. 2019. Incidence, characteristics and atherosclerotic involvement of coronary artery anomalies in adult population undergoing catheter coronary angiography. Cardiology research. 10:358-368.

Sunkara A, Chebrolu LH, Chang SM,Barker C. 2017. Coronary artery fistula. Methodist DeBakey cardiovascular journal. 13:78-80.

Turkmen S, Cagliyan CE, Poyraz F, et al. 2013. Coronary arterial anomalies in a large group of patients undergoing coronary angiography in southeast Turkey. Folia morphologica. 72:123-7.

Vasheghani-Farahani A, Kassaian SE, Yaminisharif A, et al. 2008. The association between coronary arterial dominancy and extent of coronary artery disease in angiography and paraclinical studies. Clin Anat (New York, NY). 21:519-23.

Verdini D, Vargas D, Kuo A, et al. 2016. Coronary-pulmonary artery fistulas. Journal of thoracic imaging. 31:380-390.

Wilkins CE, Betancourt B, Mathur VS, Massumi A, et al. 1988. Coronary artery anomalies: a review of more than 10,000 patients from the Clayton Cardiovascular Laboratories. Texas Heart Institute Journal. 15:166-73.

Yamanaka O, Hobbs RE. 1990. Coronary artery anomalies in 126,595 patients undergoing coronary arteriography. Catheterization and cardiovascular diagnosis. 21:28-40.

Yaymaci B, Ugurlucan M, Basaran M, et al. 2010. Solitary coronary artery nourishing the entire heart. Ann Saudi Med. 30:81-3.

Yildiz A, Okcun B, Peker T, et al. 2010. Prevalence of coronary artery anomalies in 12,457 adult patients who underwent coronary angiography. Clinical cardiology. 33: E60-4.

Yuksel S, Meric M, Soylu K, et al. 2013. The primary anomalies of coronary artery origin and course: a coronary angiographic analysis of 16,573 patients. Experimental \& Clinical Cardiology. 18: 121-3. 